\title{
Symbolic-Numeric Computation
}

Dongming Wang

Lihong Zhi

Editors 


\section{Contents}

On a Generalized Companion Matrix Pencil for Matrix Polynomials

Expressed in the Lagrange Basis

Robert $M$. Corless

Ill-conditioned Properties and Hybrid Computations Matu-Tarow Noda

Rational Interpolation and Its Ill-conditioned Property Hiroshi Kai

Computing Approximate GCD of Multivariate Polynomials Masaru Sanuki

Structured Low Rank Approximation of a Sylvester Matrix Erich Kaltofen, Zhengfeng Yang and Lihong Zhi

Implementation of Fast Low Rank Approximation of a Sylvester Matrix . . . . 85 Bingyu Li, Zhuojun Liu and Lihong Zhi

New Algorithms for Exact and Approximate Polynomial Decomposition .... 99 Mark Giesbrecht and John May

Amortized Bound for Root Isolation via Sturm Sequences Zilin Du, Vikram Sharma and Chee K. Yap

An Algorithm of Real Root Isolation for Polynomial Systems with Applications to the Construction of Limit Cycles Zhengyi Lu, Bi He, Yong Luo and Lu Pan

An Algebraic Method for Separating Close-Root Clusters and the

Minimum Root Separation Tateaki Sasaki and Fujio Kako

On the Location of Zeros of an Interval Polynomial

Hiroshi Sekigawa and Kiyoshi Shirayanagi

Root-Finding with Eigen-Solving

Victor Y. Pan, Dmitriy Ivolgin, Brian Murphy, Rhys Eric Rosholt, Yuqing Tang, Xinmao Wang and Xiaodong Yan

Galois Theory via Eigenvalue Methods 211

David A. Cox 
Pythagore's Dilemma, Symbolic-Numeric Computation, and the Border

Basis Method

Bernard Mourrain

Proposal for the Algorithmic Use of the BKK-Number in the Algebraic

Reduction of a 0-dimensional Polynomial System ................... 245

Hans J. Stetter

Evaluation of Jacobian Matrices for Newton's Method with Deflation

to Approximate Isolated Singular Solutions of Polynomial Systems ....... 269

Anton Leykin, Jan Verschelde and Ailing Zhao

On Approximate Linearized Triangular Decompositions ................279

Marc Moreno Maza, Greg J. Reid, Robin Scott and Wenyuan Wu

On the Extended Iterative Proportional Scaling Algorithm

Ming-Deh Huang and Qing Luo

Preprocessing for Finite Element Discretizations of Geometric Problems ... 315 Hong Gu and Martin Burger

Symbolic Computation Sequences and Numerical Analytic Geometry

Applied to Multibody Dynamical Systems

Wenqin Zhou, David J. Jeffrey and Greg J. Reid

A Symbolic-Numeric Approach to an Electric Field Problem

David J. Jeffrey, Silvana Ilie, James M. Gardiner

and Steven W. Campbell

Financial Applications of Symbolically Generated Compact Finite

Difference Formulae

Jichao Zhao, Robert M. Corless and Matt Davison

Symbolic Analyzer for Large Lumped and Distributed Networks

Majid A. Al-Taee, Fawzi M. Al-Naima and Bessam Z. Al-Jewad

Author Index 